\title{
TRATAMIENTO DE LOS TRASTORNOS DE LA CONDUCTA Y DE LA EPILEPSIA EN LA PARALISIS CEREBRAL INFANTIL
}

\author{
Dra. ALICTA PADILLA
}

Serpicio de Neuropsiquiatría. Hospital "Roberto del Rio". Santiago.

Estos tipos de perturbaciones, al igual que otras que se acaban de exponer, no son acompañantes obligados de la parálisis cerebral, pero sí frecuentes.

Nos referiremos primero a los trastornos de la conducta. Como todas las otras perturbaciones acompañantes del trastorno motor, pueden ser las únicas o estar asociadas a otras, que las modifican $y$ complican. Además pueden tener diversos grados de intensidad.

Aquí también los casos leves interesan poco pues al no constituir especial problema no necesitan tampoco ser afrontados con mucha actividad. Son Ios de mayor envergadura los que deben preocuparnos. Es una observación ya bastante conocida que las incapacidades físicas son desfavorablemente influídas por los factores emocionales traumatizantes al interferir la terapéutica física.

Existen casos en que la terapia fisica falló hasta que se instituyó un tratamiento siquítrico. Pero aquí no nos vamos a referir a los tratamientos siquiátricos de fondo de los casos más graves, que en verdad deben quedar en manos del especialista en neurosiquiatría y que afortunadamente no son los más corrientes. Nos referiremos sólo a los casos más simples. Aquí, quizás, más que en otros aspectos de la medicina, vale más prevenir que curar.

Las bases de Ia conducta que el médico debe seguir en este sentido, se comprenderán mejor si tenemos presente que el origen de las perturbaciones de la conducta en los niños con parálisis cerebral se encuera en Ios siguientes factores, que actúan aisladamente 0 , lo que es tal vez más frecuente, en combinaciones:

1. El daño orgánico de ciertas estructuras del S.N.C.

2. La reacción inadecuada del ambren. te con respecto al niño y su incapacidad.

3. La reacción propia del niño frente a la enfermedad.

E1 extendernos en los detalles de cada uno de estos factores nos llevaría a colocarnos fuera del tema específico que aquí vamos a desarrollar.
Pero, no es posible, en realidad, hablar de tratamiento de la conducta sin hacer mención a ellos. (Se nos perdonará, pues, si caemos en repeticiones de lo que otros relatores ya han dicho, pero las reduciremos a lo indispensable). No debe perderse de vista, por lo demás, que la acción terapéutica que debamos realizar es más de orden preventivo que curativo, en lo referente a los factores 2 y 3 .

Como se comprende, las anomalías conductuales dependientes del daño orgánico cerebral son escasamente modificadas por los tratamientos médicos, pero pueden ser parcialmente compensadas a anuladas con medidas de orden pedagógico o de reajustes ambientales que se confunden con las medidas que se emplean en los otros factores. Aquí el uso de medicamentos (tranquilizadores, timolépticos) es una vía promisoria, que en ciertos casos ya nos han dado buenos resultados, pero son muchísimo más modestos que lo que la propaganda de los fabricantes nos quiere hacer aceptar. Ojalá que en un futuro no muy lejano podamos decir lo contrario.

Las consecuencias que el daño cerebral tiene sobre la inteligencia $y$ en las funciones que intervienen en el aprendizaje, traducen en dificultades escolares de todo orden que ya han sido tratadas por la Dra. Riquelme. Las repercusiones de este mismo daño sobre el carácter, el temperamento y la personalidad, se confunden, en parte, con manifestaciones semejantes desencadenadas por los otros dos factores y como éstas son en mayor o menor medida susceptibles de prevención y de modificación mediante medidas sicopedagógicas y sicoterápicas, nos referiremos con más extensión a ellas. Sin embargo, conviene que recordemos que el daño cerebraI en sí puede acarrear las siguientes características: apego a la actividad concreta, sin grandes variaciones, de lo que resulta perseveración en las acciones: fatigabilidad, que lleva a rendimientos menores; dificultad de adaptación a las situaciones nuevas, que conduce a fracasos, con la consiguiente ansie- 
dad que puede llevar hasta las llamadas "reacciones catastróficas", (sensación angustiosa de insuficiencia, de que todos los caminos están cerrados, de que no hay como escapar a una catástrofe); cambios bruscos del humor; baja tolerancia a las frustraciones. Del conjunto de estos rasgos resulta un apego especial de estos niños a las rutinas, pero cuando logran superar una dificultad o situación nueva, se pueden entusiasmar de tal manera que se concentran en la nueva tarea o estímulo, recayendo en una nueva rutina. El objeto de recordar todo esto en este momento es el llamar Ia atención hacia el hecho de que cuando estos rasgos se presenten en un niño con parálisis cerebral no debe pensarse con ligereza de que sean re. accionales psicógenos y se pretenda tratarlos como tales. Pero sí que debe adoptarse una política que evite las frustraciones y fracasos excesivos y se vaya paulatinamente acostumbrando al niño a salir de las rutinas.

Mucho mayor efectividad tendrán las medidas que se toman para evitar el otro tipo de perturbaciones conductuales de estos nin̄os, o sea, las que representan reacciones psicógenas dē niño a la actitud del ambiente o a su propias anomalías. Haremos a continuación un somero análisis del mecanismo de producción y de presentación de dichas perturbaciones, para comprender las medidas que deban tomarse para evitar su desarrollo y para impedir un mayor agravamiento si ya están presentes o tratar de eliminarlas en mayor o menor grado. Se confunden, pues, en parte Ia acción dirigida a prevenir y la acción dirigida a contrarrestar o eliminar las perturbaciones ya producidas.

Es básica la normal relación con la familia para la madurez emocional y después para la verdadera independencia. De la familia obtendrá el niño su primera idea de como la gente piensa y se conduce con respecto a él.

En nuestros casos la familia y principalmente los padres están emocionalmente alterados, tienen temores y ansiedades; el diagnóstico, la naturaleza de la enfermedad que altera el desarrollo normal, la iniciación del tratamiento, incluso de la preocupación económica contribuyen a este estado de perturbación emocional de los padres.

Los problemas conyugales se intensifican o aparecen en torno al niño, a veces en relación con la presunta herencia de la enfermedad de uno $u$ otro de los padres.

Repetidas frustraciones $\mathbf{y}$ esperanzas perdidas producen cierto retraso del niño, por los padres quienes desarrollan sentimientos de culpa y de haber sido castigados. Reaccionan a esto sobreprotegiendo $e$ infantilizando al niño y más amor, que causa confusión e inseguridad en el niño.

Estas actitudes de los padres provocan desviaciones del desarrollo psicológico normal del nin̄o. Si priman las actitudes de rechazo, encontraremos nin̄os con tendencia al aislamiento, que tratan de evitar el contacto con la realidad, toman fre cuente refugio en la fantasia, tienen tendencia a defenderse a sí mismos, colo cando la culpa en otra persona, o transfieren su malestar respecto a sus incapacidades en el resto de la gente llegando a ser hostiles al ambiente.

Si están sobreprotegidos desarrollan una exagerada preocupación de sí mismos y dan poca importancia al ambiente $y$ a los demás. Por su trastorno motor uni. al acostumbramiento a que otros reali. zan los actos necesarios para satisfacer sus necesidades tienen excesiva tendencia a la pasividad y falta de iniciativa.

Tienen menor número de intereses que los normales de su edad y en las frustraciones manifiestan tendencia a hacer expresiones emocionales infantiles, incontroladas.

Todas estas actitudes inadecuadas se deben en general, a que los padres son incapaces de aceptar la realidad de la situación, las limitaciones físicas y especialmente las mentales del niño, y en consecuencia desarrollan esperanzas o ambiciones respecto a él, que no será más tarde capaz de realizar y lo harán sentirse más insatisfecho.

De lo que se acaba de decir se deducen fácilmente las normas para prevenir y afrontar las perturbaciones de este orden.

Es necesario en primer lugar dar a los padres una clara y prolija información sobre la enfermedad, su origen, su desarrollo y sus posibilidades, el grado de la incapacidad motora, la mejoría que es posible obtener con el tratamiento y la imposibilidad de precisar el tiernpo que demoren en aparecer los resultados.

Es de primordial importancia llevar a los padres y a la familia a aceptar la condición desventajosa que existe, que el ni- 
no sea aceptado como es, sus incapacidades honradamente comprendidas. Esto ayudará más tarde a la aceptación de su enfermedad por el niño mismo, a quien paulatinamente debe hacerse comprender sus limitaciones, pero a la vez sus capacidades que deben ser apreciadas $y$ estimuladas.

Evitar de esta forma la actitud supersolícita que limita al niño su experiencia con el mundo externo, y que hace que sea considerado más infantil de lo que le corresponde, que sea catalogado como un ser incapaz de comprender, en cuya presencia sus padres llegan a veces a discutir los problemas creados por su misma enfermedad.

Nos hemos referido primero, al primer ambiente natural del nin̄o que son sus padres. Parecidas consideraciones valen para el resto del ambiente familiar, especialmente para los hermanos que se sienten confusos e inbibidos frente a un nino que no puede tomar igual parte en los juegos, a quien no se pueden dar las mismas responsabilidades y que necesita de ayuda. Su reacción será como sea la de sus padres. El resto de la familia debe conocer la actitud que deben tomar y las responsabilidades que les caen.

Las amistades y el ambiente en general aceptarán al niño conforme a la forma en que es aceptado por su familia.

Más tarde, en relación al ambiente escolar, si su condición le permite asistir a la escuela, se crean dificutlades a las que, sólo nos referiremos muy brevemente pues ya han sido tratadas por la Dra. Riquelme.

En esta etapa la pesquiza de alteraciones de la vision, audición y lenguaje, es esencial, si no se han puesto de manifiesto antes de por sí.

Promover una estrecha relación entre profesor y alumno es indispensable para obtener rendimientos satisfactorios.

En su relación con el ambiente a lo largo de su desarrollo, en cualquier etapa, será siempre esencial evitar la piedad a fin de que se desarrolle en el niño un sentimiento de confianza en sí mismo y de autodeterminación.

Sobre la reacción del niño mismo frente al ambiente $y$ a sus propias anomalias, para comprenderlas, debemos pensar en sus capacidades. Sus limitaciones motoras le impiden tener el mismo contacto físico con el ambiente que el niño normal lo que determina que sus primeras expe- riencias y vivencias de este nuevo medio sean diferentes. Asi, entonces, fácilmente puede llegar a la sensación de su propio desvalimiento.

Por otro lado tiene las mismas necesidades que los niños sanos: ser querido, querer, sentirse seguro, sentirse deseado, necesidad de autoestimación, oportunidad de expresarse, sentimiento de autosuficiencia.

Estas necesidades unidas a su incapacidad relativa y a las posibles reacciones del ambiente de rechazo o sobreprotección se traducen en niños más inseguros, con más temores y más sentimientas de culpa que los normales de su edad.

El primer año de vida es decisivo en el desarrollo de la personalidad. Muchos estudios sobre este punto indican que la falta de amor $y$ de estrechas relaciones humanas impiden el normal desarrollo psicológico.

Se da con justificada razón gran importancia a la relación madre-hijo en esta etapa.

La ausencia de una relación armoniosa hará sentir al niño más tarde o más temprano, que está solo, incluso que vive en mundo hostil.

La relación normal por el contrario le dará seguridad lo que evitará que más tarde tenga una actitud defensiva $\mathbf{u}$ hostil hacia su medio o que se retire a su fantasía para aislarse de un mundo que queda más allá de su comprensión.

Aparte de las necesidades afectiva básicas que el recién nacido normal debe en. contrar al llegar al mundo tiene también necesidades básicas físicas: alimentos, abrigo, bienestar. Si no está satisfecho en ellas se torna ansioso, tenso, inseguro, llora.

Ya en este momento tiene el niño con parálisis cerebral una gran desventaja. Frecuentemente no puede succionar satisfactoriamente del pecho de su madre, además su hipertonía e hiperexcitabilidad le impide la normal y acogedora estadia en brazos de la madre. El niño en esta forma carece de la sensación táctil y kinestésica de ser protegido.

Paciencia infinita $\mathrm{y}$ un afecto profundo por el niño pueden atenuar u obviar esta desventaja.

Este problema de dar los alimentos es básico y puede desarrollarse de una u otra manera en cualquier edad.

Los consejos que en estos casos ayudarán a superar las dificultades son: En el 
lactante: El niño debe estar con hambre en el momento de mamar, seco, ni demasiado frío ni excesivamente abrigado, en una posición cómoda y despierto, esto último en relación a que a veces horarios demasiado rígidos hacen que el niño sea interrumpido en su sueño para colocarlo al pecho, debe ser mantenido erguido despues de mamar para expulsar el aire deglutido, incluso esto puede ser necesario hacerlo varias veces durante la toma del alimento.

En cualquier edad requerirá la alimentación de una gran dosis de paciencia, una atmósfera tranquila, libre de ruidos violentos, que no revele apuro. Introducción paulatina de alimentos nuevos y si es posible la asociación de alimentos con otras sensaciones agradables. Más adelante una actitud flexible en relación al modo de comer del niño, dando sjempre oprtunidad para que el acto de comer sea un acto agradable.

En el niño con serios problemas motores estas normas deben ser extremadas y dependen mucho de la actitud de la madre.

Igual ocurre en la satisfacción de cualquiera de las otras de sus necesidades fisiológicas.

Como estos niños requieren ayuda física, permanenecen o tienen que ser tomados en brazos un mayor tiempo que los normales, si este acto se realiza en forma brusca y repentina les produce una sensación verdaderamente terrorífica. Igual ocurre con la presencia brusca de un adulto que se acerque, tenga que tomarlo o manipulear alguna parte de su cuerpo.

Si el niño tiene que ser llevado en brazos, esto siempre debe ser hecho en forma suave y considerada, ya que por haber tenido menor oportunidad de independencia son sensitivos e hiperreactivos. Cuando son más grandes y pueden comprender, debe explicárseles en cada oportunidad lo que va a hacerse con ellos, es decir en ningún momento el niño debe ser transportado como un objeto inanimado sino como una persona.

Otro medio de vital importancia en el contacto y expresión, el habla, ha sido ya tratado en el trabajo de la Dra. Astorga. Sólo diremos que la hipersolicitud en satisfacer sus deseos no estimula su desarrollo, por otra parte la exigencia más allá de sus capacidades provocará tensión.

Lo importante no es apreciar Ia claridad del lenguaje sino tratar que el nin̄o se sienta comprendido en sus voces o signos que emplea para señalar determinado objeto o acto.

Las actividades de auto-ayuda; alimentarse, vestirse escribir, etc. dentro de sus posibilidades deben ser enseñadas, con la paciencia y lentitud necesarias, de acuerdo con su capacidad y no con la edad en que los normales lo harían. A ellas ha hecho referencia la Sra. Pedraza.

Especial interés requiere el desarrollo del control de los esfinteres, que será tardio y lento es establecerse, dependiendo del grado de incapacidad motora y mental. El forzar esta función y establecer normas más o menos rígidas para obtenerlas será altamente perjudicial y contraproducente, pudiendo llevarlo a distanciarse de los hábitos de limpieza o, al contrario, a una meticulosidad excesiva.

Cuando al niño enfermo ya crecido haya que transportarlo para estas funciones, se agregan factores psíquicos de importancia que dificultan el aprendizaje, que se ponen especialmente en evidencia si esta ayuda que tiene que prestar la madre la irrita en contra del niño, o por atras preocupaciones no puede atenderio en el momento que él lo solicita.

Una silla especial para estos hábitos de toilette pueden ser de gran ayuda, le dan mayor seguridad física $\mathrm{y}$ más independencia.

Si recordamos la tendencia que pueden tener estos niños a reacciones infantiles en sus frustraciones daremos la debida importancia de los detalles del control de los esfínteres.

Pasamos ahora 1 tratamiento de la epilepsia en la parálisis cerebral.

Las formas convulsivas de epilepsia (limitadas a un segmento del cuerpo $o$, más frecuentemente, generalizadas) acompañan a la parálisis cerebral en un porcentaje que oscila, según los autores, entre un 24 y un $46 \%$ de los casos. También pueden coexistir otras formas de manifestaciones comiciales, pero son bastante más raras. Es justificado aceptar que prácticamente en todos los casos se trata de una epilepsia secundaria a las mismas lesiones que producen los trastornos motores y las demás perturbaciones de la parálisis cerebral. 
Por ahora, sólo en un reducido número de casos está indicado un tratamiento quirúrgico y no nos corresponde hacerlo, como porque, en todo caso, cae en la esfera de acción de un centro muy especializado. Sin embargo, no está de más que recordemos que una de las indicaciones más definidas para una hemisferectomía es, justamente, la coexistencia de epilepsia, hemiplegia y retardo mental grave.

Prácticamente en todos los casos debe instituirse un tratamiento médico como el que se lleva a efecto en cualquiera epilepsia. Se usarán los medicamentos anticonvulsivantes junto a otros medicamentos coadyuvantes $y$ otras medidas de orden general.

\section{RESUMEN}

TRATAMIENTO DE LOS TRASTORNOS DE LA CONDUCTA Y LA EPILEPSIA EN LA PARÁLISIS CEREBRAL INFANTIL

Se revisa, de acuerdo con los conocimientos actuales, el tratamiento de los trastornos de conducta y de la epilepsia en niños con parálisis cerebral.

En relación a los trastornos de conducta, se analiza su mecanismo de desarrollo, considerando su origen en 3 factores:

1.' Daño orgánico de ciertas estructuras deI S.N.C.

$2^{\circ}$ Reacción inadecuada del ambiente con respecto al niño y su incapacidad.

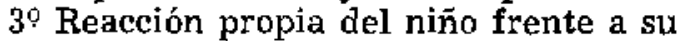
enfermedad.

Se hace notar la diferencia de reacción al tratamiento de las perturbaciones ocasionadas por el daño cerebral, de aquellas que representan reacciones sicógenas.

Se dan las indicaciones terapéuticas y preventivas aplicables en cualquier medio no especiailzado.

En relación a la epilepsia, se hace referencia a las normas habituales de tratamiento del Servicio.

\section{SUMMARY}

THE TREATMENT OF THE BEHAVIOR DISORDERS AND OF THE EPILEPSY IN THE INFANTIE CEREBRAL PALSY

The present state of our knowledge of the treatment of behavior disorders and of the epilepsy in children with cerebral palsy is surveyed.
With regard to the behavior disorders, their developmental mechanism is analyzed, three factors being taken into account as their origin:

1) the organic injury of certain structures of the central nervous system;

2) the inadquate reaction of the environment to the child and his limited intelligence;

3 ) the reaction of the child himself to his disease.

Attention is paid to the difference between the response to the treatment of the disturbances caused by the cerebral injury, and that to the treatment of the disturbances which denote psychogenie reactions.

Therapeutic and preventive indications which may be applied in every not specialized environment, are given.

With regard to the epilepsy, the habitual therapeutic patterns of the Service are referred to.

\section{ZUSAMMENFASSUNG}

DIE BEHANDLUNG DER STÖRUNGEN IM BENEHMEN UND DER EPILEPSTE BEI DER ZEREBRALEN LAEHMUNG DES KINDES

Ueber den gegenwärtigen Stand der Kenntnisse von der Behandlung der Störungen im Benehmen und der Epilepsie bei der zerebralen Lähmung des Kindes wird ein Ueberblick gegeben.

In Bezug auf die Störungen im Benehmen wird der Mechanismus ibrer Entwicklung näher betrachtet und ihr Ursprung in drei Faktoren erblickt:

1) die organische Schädigung bestimmter Strukturen des Zentralnervensystems;

2) die dem Kind und seiner geistigen Beschränktheit nicht Rechnung tragende Reaktion der Ungebung;

3) die eigene Reaktion des Kindes angesichts seiner Erkrankung.

Auf den Unterschied zwischen der Antwort auf die Behandlung der durch den Hirnschaden verursachten Störungen und der Antwort auf die Behandlung der psychogene Reaktionen darstellenden Störungen wird hingewiesen.

Für die Behandilungs- und VorbeugungsMassnahmen, die in jeder nicht spezialisierten Umgebung anwendbar sind, werden die Indikationen angegeben.

Betreffs der Epilepsie wird auf die in der Krankenabteilung üblichen Normen der Behandlung Bezug genommen. 